\title{
Adaptive Generalized Selection Combining (A-GSC) Receivers
}

\author{
Athanasios S. Lioumpas, Student Member, IEEE, George K. Karagiannidis, Senior Member, IEEE, \\ and Theodoros A. Tsiftsis, Member, IEEE
}

\begin{abstract}
We introduce an adaptive generalized selection combining (A-GSC) receiver that can be efficiently applied in diversity rich fading environments such as in ultra-wideband applications. The branches that participate in the combining process are selected taking into account the quality of both the total combining output signal-to-noise ratio (SNR) and the individual SNR of each branch. The proposed scheme achieves better adaptation to channel conditions compared to other competing schemes such as normalized threshold GSC (NT-GSC), minimum selection GSC (MS-GSC) or minimum estimation and combining GSC (MEC-GSC), with no further complexity. The A-GSC receiver compromises between the power consumption, hardware complexity and performance gain that each additional diversity branch induces, resulting in significant savings in power and computational resources.
\end{abstract}

Index Terms-Diversity techniques, fading channels, generalized selection combining (GSC).

\section{INTRODUCTION}

M ULTICHANNEL receivers followed by certain diversity combining techniques can significantly improve the performance of wireless communication systems and are of great interest even more in the emerging broadband communication systems (e.g. ultra-wideband (UWB)), where the number of diversity paths can be considerably large due to the strong multipath effects.

The optimal diversity combining scheme is the well-known maximal ratio combining (MRC) [1]- [3], which attains the highest output signal-to-noise ratio (SNR) of any combining scheme, independently of the distribution of the branch signals. The performance gain of MRC comes at the cost of increased hardware complexity and power consumption since all the available signal replicas are combined. However, in designing practical communications systems for diversity rich environments, the receiver's complexity and power consumption play an important role, leading to an inevitable compromise between complexity and system performance.

Among the low-complexity combining schemes for diversity rich environments, generalized selection combining (GSC), also known as hybrid-selection MRC (H-S/MRC), was the first to be proposed [4]- [6]. In the conventional GSC receiver the number of combined diversity branches is predetermined, i.e. the $L_{c}$ branches (among $L$ available ones) with the highest SNR are selected and combined as per the

Manuscript received October 2, 2007; revised February 1, 2007 and May 9, 2008; accepted July 13, 2008. The associate editor coordinating the review of this paper and approving it for publication was I. Collings.

The authors are with the Wireless Communications Systems Group (WCSG), Electrical and Computer Engineering Department, Aristotle University of Thessaloniki, 54124 Thessaloniki, Greece (e-mail: \{geokarag, alioumpa, thtsif\}@auth.gr).

Digital Object Identifier 10.1109/T-WC.2008.071087 rules of MRC. This scheme has a fixed processing complexity, while it is hard to determine a priori the number of strong diversity branches, especially in the UWB scenario, where the power delay profile (PDP) depends on the severity of scattering [7]. Moreover, the combiner may potentially discard many branches whose instantaneous SNRs are close to those branches selected, or include branches with small instantaneous SNRs. Later, a combining scheme, which was subsequently named as normalized threshold GSC (NT-GSC) [8][10], alleviated the above-mentioned problems by rejecting those branches whose instantaneous SNR does not exceed the SNR of the strongest branch multiplied by a fixed threshold. Another power saving implementation of GSC scheme was proposed in [11], termed minimum selection GSC (MS-GSC) and its performance was analyzed in [12]- [14]. With MSGSC, the receiver combines the least number of best diversity paths such that the combined SNR is above a certain threshold. In an attempt at simplifying the channel estimation complexity of these systems the output threshold GSC scheme (OT-GSC) was proposed in [15], which includes an MRC and a GSC stage. Recently, an alternative GSC receiver was proposed in [16], termed minimum estimation and combining GSC (MECGSC) (including a switching and combining (SEC) stage and a MS-GSC stage), which minimizes the average number of estimated and combined branches for a given required output SNR. As discussed so far, the alternative implementations of GSC receivers are divided into two categories: in the first one (i.e., NT-GSC), the decision on the number of combined branches is based on the SNR of each individual branch, while in the latter one the same decision is based on the combiner's output SNR (MS-GSC, OT-GSC, MECGSC). However, in NT-GSC receivers the number of combined branches is independent of the overall required quality of communication (e.g. combined SNR). In other words, the selected branches (i.e., those that satisfy the test per branch) may be unnecessarily too many, while a sufficient quality of communication could be possibly attained with less branches (e.g. in an environment with strong multipaths). On the other hand, in MS-GSC and MEC-GSC receivers the number of selected branches is determined only by the combiner's output SNR, ignoring the SNR of each individual branch. As a result, the receiver may potentially keep adding weak branches (especially in diversity rich environments with strong PDPs) in its attempt to reach the predetermined threshold, while a slightly worse or the same performance could be achieved by combining only the strong branches. The above observations lead to the conclusion that a more efficient selection of the combined branches could be achieved by taking into account both the SNR of each individual diversity branch and the 
combiner's output SNR.

In this paper, we propose an adaptive GSC receiver (AGSC), which compromises between power consumption and performance gain that each additional diversity branch induces, achieving significantly better adaptation to diversity rich fading environments, with no complexity increase. More specifically, the A-GSC receiver, keeps adding the strongest branches in order to reach the predetermined quality of communication, until it estimates that the addition of another branch does not compensate the expected performance improvement. The improved adaptation of the A-GSC scheme to variating channel conditions and the savings in power and computational resources can be significantly important in diversity rich environments (i.e. in UWB systems) where the a priori determination of the number of strong diversity branches is difficult, because of the considerably large number of diversity paths.

The remainder of the paper is organized as follows. Section II describes the A-GSC system model and mode of operation. In Section III the average number of branches is calculated for the Rayleigh fading model, while some numerical results are presented in Section IV. Finally, concluding remarks are presented in Section V.

\section{System Model AND Mode Of Operation}

Consider a multichannel diversity reception system with $L$ available branches operating in a flat fading environment, in which the receiver employs symbol-by-symbol detection. The received baseband signal over the $k$ th diversity branch in a symbol interval of duration $T_{S}$ can be expressed as

$$
r_{k}(t)=h_{k} e^{-j \varphi_{k}} s(t)+n_{k}(t)
$$

where $s(t)$ is the complex baseband information-bearing signal with average symbol energy $E_{s}, h_{k}$ is the random magnitude, $\varphi_{k}$ is the random phase of the $k$ th diversity branch gain and $n_{k}(t)$, representing the additive noise, is a zero-mean complex Gaussian random process with two-sided power spectral density $N_{0} \mathrm{~W} / \mathrm{Hz}$. The channel gains and the noise processes are assumed independent. Similarly to known GSC receivers, the proposed one is implemented in a discretetime fashion, i.e. short guard periods are periodically inserted into the transmitting signal. During these periods, the receiver performs all the necessary operations order to reach the appropriate diversity combining scheme [16].

Let $\gamma_{k}$ denote the instantaneous SNR per symbol of the $k$ th diversity branch

$$
\gamma_{k}=h_{k}^{2} \frac{E_{s}}{N_{0}}, \quad k=1, \ldots, L .
$$

Further, let $\gamma_{(k)}(k=1, \ldots, L)$ represent the instantaneous branch SNRs per symbol in descending order, that is, $\gamma_{(1)}>$ $\gamma_{(2)}>\ldots>\gamma_{(L)}$.

By implementing the A-GSC technique, the receiver tries to raise the combined SNR, $\gamma_{c}$, above the threshold, $\gamma_{T}$, by gradually changing from a low order GSC scheme (i.e. a GSC with small number of combined active branches) to a higher order GSC scheme. We note that $\gamma_{T}$ determines the

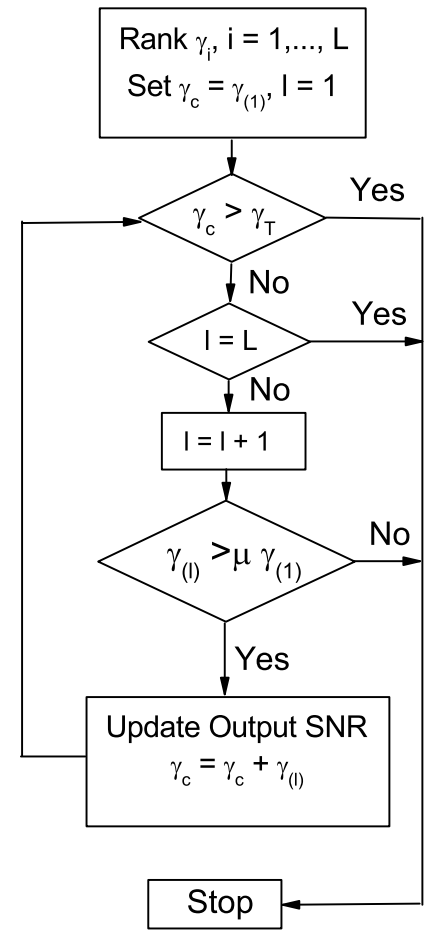

Fig. 1. A-GSC receiver : Mode of operation.

overall required quality of communication, usually adjusted dynamically according to the service that is used. However, the threshold $\gamma_{T}$ is not the only criterion that determines the number of the combined diversity branches; as soon as the receiver estimates that the addition of another branch does not compensate the expected performance improvement, it stops the selection process and combines the selected branches as per the rules of MRC.

The mode of operation of the A-GSC scheme is described in Fig.1. In particular, the receiver first estimates the channel gains and phases for all the diversity paths. Then, starting from the best path, the receiver tries to increase the combined SNR $\gamma_{c}$ above the threshold $\gamma_{T}$ by adding more diversity branches unless the SNR of the next branch is below the value $\mu \gamma_{(1)}$. More specifically, if $\gamma_{(1)}>\gamma_{T}$, then only one branch will be used. Otherwise, the receiver will test the SNR of the next strongest branch (i.e. $\gamma_{(2)}$ ) against the value $\mu \gamma_{(1)}$. If $\gamma_{(2)}<$ $\mu \gamma_{(1)}$ then the selection process terminates and the second branch is rejected, i.e. an $L$-branch SC combiner is used. If $\gamma_{(2)}>\mu \gamma_{(1)}$ the receiver adds the SNR of those two branches, i.e. $\gamma_{c}=\gamma_{(1)}+\gamma_{(2)}$ and tests it against the output threshold $\gamma_{T}$. This procedure is continued until either the combined SNR is above $\gamma_{T}$, or the SNR of a single branch is below $\mu \gamma_{(1)}$, or all $L$ branches are activated. In other words, the selection process terminates, if the combined SNR after the activation of the first $l$ branches is above the threshold, or the ratio of the SNR of the $(l+1)$ th branch to that of the first branch is below the required threshold $\mu \in[0,1]$.

The above features constitute the power saving of A-GSC over NT-GSC and MS-GSC: by using the A-GSC scheme we 


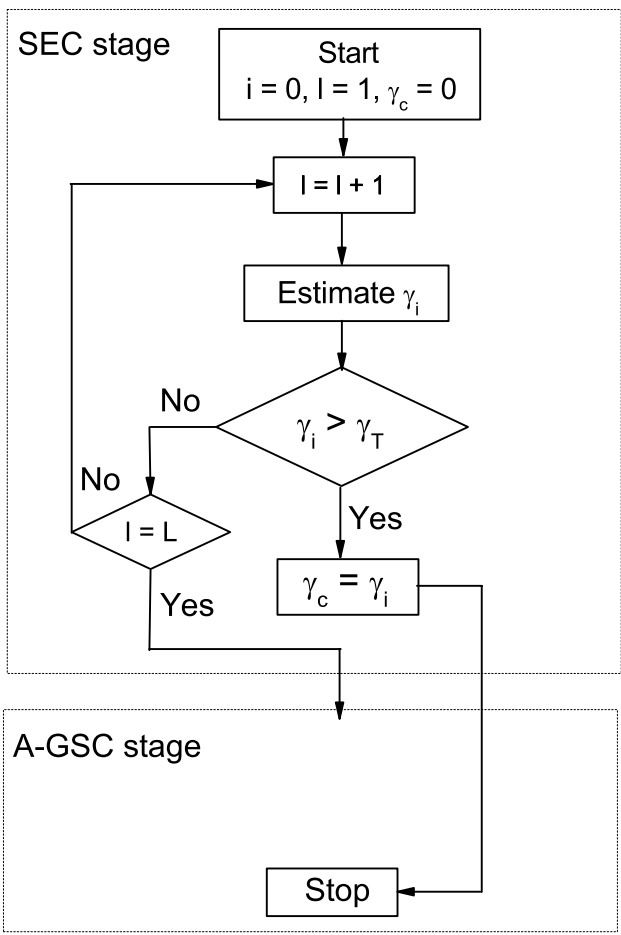

Fig. 2. A-GSC receiver with a SEC stage : Mode of operation.

can avoid either the unnecessary combining of many strong branches that a threshold per branch may induce, or the unnecessary combining of many weak branches that may occur by applying a threshold at the combined SNR. The improved adaptation of the A-GSC scheme to channel conditions and the corresponding power saving can be significantly important in diversity rich environments (i.e. in UWB systems) where the a priori determination of the number of strong diversity branches is difficult, since the number of diversity paths can be considerably large characterized by a variety of PDPs that depend on the severity of scattering.

The A-GSC scheme can be also applied to MEC-GSC receivers by substituting the MS-GSC stage with the A-GSC one, minimizing in this way the number of estimated branches. The flow chart in Fig.2 describes the mode of operation of a A-GSC receiver with a SEC stage. More specifically, the receiver starts by estimating sequentially through the $L$ available branches until one branch SNR exceeds the required output SNR $\gamma_{T}$. If the SNR of one branch is above the threshold, then this branch is used for data detection and the selection process is terminated. If none of the $L$ branches meet the desired threshold during this SEC stage, then the receiver switches to the A-GSC stage described above. A-GSC receivers do not increase the hardware complexity, compared to the other GSC receivers, since they require the same number of channel estimations (i.e. same number of radio frequency chains, matched filters, etc.); the power savings arise from the reduced number of active branches at the combining stage, induced by the different selection algorithm.

\section{Average Number of Combined BRAnCHes}

Since the importance of the GSC systems lies in the fact that they reduce the number of combined branches compared to the optimal MRC systems, it is important to study the average number of combined branches of the A-GSC receivers as a quantification of the power savings. The analysis that follows is valid for both the A-GSC scheme and the A-GSC with the SEC pre-stage, since the MS-GSC and MEC-receivers have exactly the same number of combined branches [16].

Assume that $\gamma_{k}$ 's are $L$ nonnegative random variables (rvs) with probability density function (pdf), $p_{\gamma_{k}}(x)$ and cumulative distribution function (cdf), $P_{\gamma_{k}}(x)$. Depending on the fading conditions, the number of active MRC branches with A-GSC, denoted by $N_{c}$, takes value from 1 to $L$. We also represent the sum of the $l$ largest SNRs, i.e. $\sum_{i=0}^{l} \gamma_{(i)}$, as $\Gamma_{l}$. Moreover, $M_{(l / L)}$ and $N_{(l / L)}$ stand for the events that a MS-GSC or a NT-GSC is used respectively that employ the $l$ strongest branches out of $L$ available ones, while $M_{\left(l^{+} / L\right)}$ and $N_{\left(l^{+} / L\right)}$ denote the events that the MS-GSC or NT-GSC use $i>l$ branches. The average number of branches combined by the A-GSC can be calculated as a function of the probabilities of the above events. From the mode operation of the A-GSC, the probability that $N_{c}$ branches are combined can be calculated as follows.

$$
\begin{gathered}
\operatorname{Pr}\left[N_{c}=1\right]=\operatorname{Pr}\left[M_{(1 / L)} \text { OR }\left(N_{(1 / L)} \text { AND } M_{\left(1^{+} / L\right)}\right)\right] \\
\operatorname{Pr}\left[N_{c}=2\right]=\operatorname{Pr}\left[\left(M_{(2 / L)} \operatorname{AND~} N_{\left(1^{+} / L\right)}\right)\right. \\
\left.\operatorname{OR}\left(N_{(2 / L)} \text { AND } M_{\left(2^{+} / L\right)}\right)\right] \\
\vdots \\
\operatorname{Pr}\left[N_{c}=l\right]=\operatorname{Pr}\left[\left(M_{(l / L)} \text { AND } N_{((l-1)+/ L)}\right)\right. \\
\left.\operatorname{OR}\left(N_{(l / L)} \text { AND } M_{\left(l^{+} / L\right)}\right)\right] \\
\left.\operatorname{Pr}\left[N_{c}=L\right]=\operatorname{Pr}\left[N_{(L / L)} \text { AND } M_{\left((L-1)^{+} / L\right)}\right)\right]
\end{gathered}
$$

The probabilities that MS-GSC selects either a single branch, or, $l$, or $L$ branches are given respectively by [14]

$$
\begin{aligned}
\operatorname{Pr}\left[M_{(1 / L)}\right] & =\operatorname{Pr}\left[\Gamma_{1} \geq \gamma_{T}\right]=1-P_{\Gamma_{1}}\left(\gamma_{T}\right) \\
\operatorname{Pr}\left[M_{(l / L)}\right] & =\operatorname{Pr}\left[\Gamma_{l-1}<\gamma_{T} \text { AND } \Gamma_{l} \geq \gamma_{T}\right]= \\
& =P_{\Gamma_{l-1}}\left(\gamma_{T}\right)-P_{\Gamma_{l}}\left(\gamma_{T}\right) \\
\operatorname{Pr}\left[M_{(L / L)}\right] & =\operatorname{Pr}\left[\Gamma_{l-1}<\gamma_{T}\right]=P_{\Gamma_{l-1}}\left(\gamma_{T}\right)
\end{aligned}
$$

where $P_{\Gamma_{l}}(\cdot), l=1, \ldots, L-1$, is the cdf of the sum of the $i$ th order statistics among $L$ RVs. Additionally, the probability that $i>l$ branches are selected, equals to

$$
\left.\operatorname{Pr}\left[M_{\left(l^{+} / L\right)}\right)\right]=\operatorname{Pr}\left[\Gamma_{l}<\gamma_{T}\right]=P_{\Gamma_{l}}\left(\gamma_{T}\right)
$$

Furthermore, by using [17, eq. (5)], the probability that a NTGSC receiver selects the $l$ strongest branches can be written as

$\operatorname{Pr}\left[N_{l / L)}\right]=\operatorname{Pr}\left[\gamma_{(l)} \geq \mu \gamma_{(1)}>\gamma_{(l+1)}\right]=F_{l}(\mu)=$
$l\left(\begin{array}{c}L \\ l\end{array}\right) \int_{0}^{\infty} p_{\gamma}(x)\left[P_{\gamma}(\mu x)\right]^{L-l}\left[P_{\gamma}(x)-P_{\gamma}(\mu x)\right]^{l-1} d x$

where $\left(\begin{array}{l}L \\ l\end{array}\right)$ denotes the binomial coefficient. The probability that $i>l$ branches are employed by the NT-GSC receiver is 
given by

$$
\begin{aligned}
& \left.\operatorname{Pr}\left[N_{\left(l^{+} / L\right)}\right)\right]=1-\operatorname{Pr}\left[\mu \gamma_{(1)}>\gamma_{(2)} \quad \text { OR } \ldots\right. \\
& \left.\gamma_{(l-1)} \geq \mu \gamma_{(1)}>\gamma_{(l)}\right]=1-\sum_{j=1}^{l} F_{j}(\mu), \quad l \geq 1
\end{aligned}
$$

since the events $N_{(l / L)}, l=1, \ldots, L$ are mutually exclusive (e.g. the receiver cannot use at the same time exactly two and three branches).

Finally, by taking into account the fact that the probability MS-GSC to combine $l$ branches does not affect the probability that NT-GSC combines $k$ branches [i.e. the performance (number of active branches) of the former is independent from the performance of the latter] and using (4), (5), (6) and (7), the probability that $i$ branches are combined, given by (3), reduces to

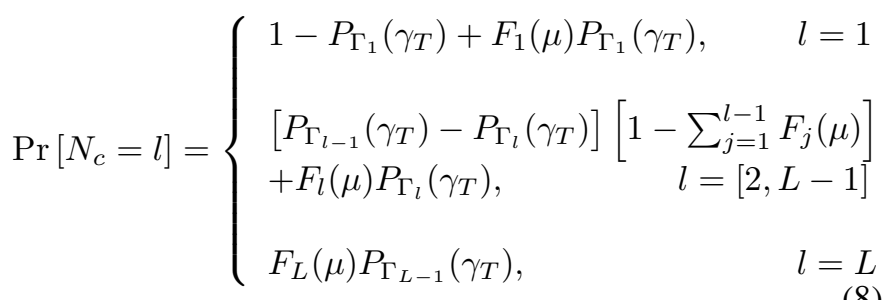

\section{A. Independent Identically Distributed Rayleigh Fading}

Suppose that $\gamma_{k}$ 's are independent and identically distributed (i.i.d) according to the common exponential distribution i.e.

$$
p_{\gamma_{k}}(x)=\frac{1}{\bar{\gamma}} e^{-\frac{x}{\bar{\gamma}}}
$$

where $\bar{\gamma}$ is the common average faded SNR. Thanks to [14], $P_{\Gamma_{i}}(\cdot)$ can be written as

$$
\begin{aligned}
P_{\Gamma_{i}}(x)= & \frac{L !}{(L-i) ! i !}\left\{1-e^{-\frac{x}{\bar{\gamma}}} \sum_{k=0}^{i-1} \frac{1}{k !}\left(\frac{x}{\bar{\gamma}}\right)^{k}+\right. \\
& \sum_{l=1}^{L-i}(-1)^{i+l-1} \frac{(L-1) !}{(L-i-l) ! l !}\left(\frac{i}{l}\right)^{i-1} \\
& \times\left[\left(1+\frac{l}{i}\right)^{-1}\left[1-e^{-\left(1+\frac{l}{i}\right) \frac{x}{\gamma}}\right]\right. \\
& \left.\left.-\sum_{m=0}^{i-2}\left(-\frac{l}{i}\right)^{m}\left(1-e^{-\frac{x}{\gamma}} \sum_{k=0}^{m} \frac{1}{k !}\left(\frac{x}{\bar{\gamma}}\right)^{k}\right)\right]\right\}
\end{aligned}
$$

while (6) reduces to

$$
\begin{aligned}
F_{l}(\mu)= & l\left(\begin{array}{c}
L \\
l
\end{array}\right) \sum_{i=0}^{L-l}(-1)^{i}\left(\begin{array}{c}
L-l \\
i
\end{array}\right) \sum_{j=0}^{l-1}(-1)^{j}\left(\begin{array}{c}
l-1 \\
j
\end{array}\right) \\
& \times 1 /[1+j+\mu(l-j-1+i)]
\end{aligned}
$$

Finally, the average number of combined paths with A-GSC can be easily obtained as

$$
\bar{N}_{c}=\sum_{i=1}^{L} i \operatorname{Pr}\left[N_{c}=i\right] .
$$

Note that for $\mu=0$, the A-GSC scheme reduces to that of MS-GSC one, while for $\gamma_{T} \rightarrow \infty$, it reduces to the NT-GSC scheme.
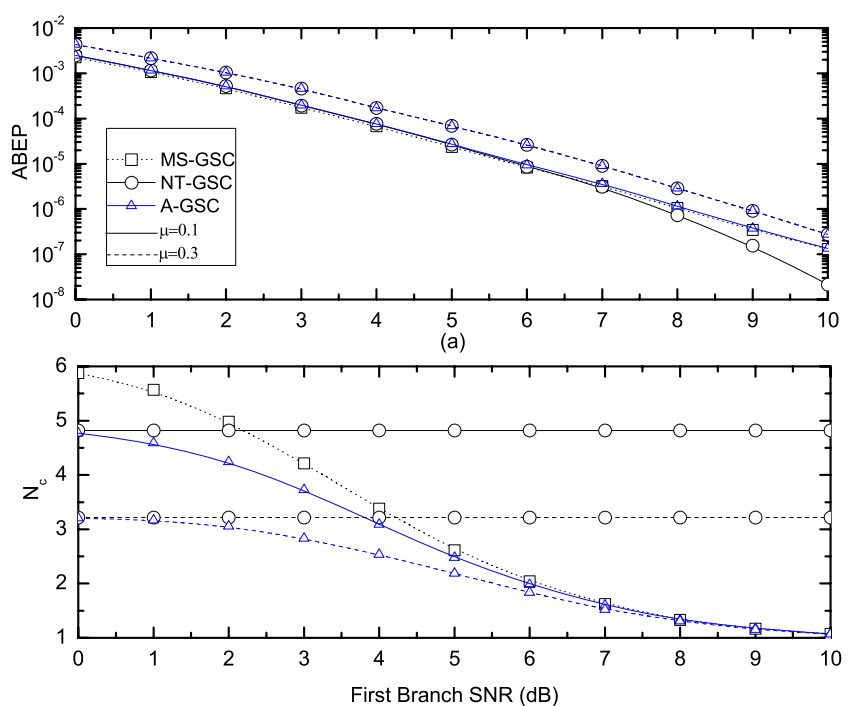

(b)

Fig. 3. ABEP and $\bar{N}_{c}$ with $L=6$ in i.i.d Rayleigh fading channels.

\section{B. Independent But Non-Identically Distributed Rayleigh Fad- ing}

For the case that $\gamma_{k}$ 's are independent but not identically distributed (i.n.i.d) nonnegative rvs with exponential pdfs

$$
p_{\gamma_{k}}(x)=\frac{1}{\bar{\gamma}_{k}} e^{-\frac{x}{\bar{\gamma}_{k}}}
$$

where $\bar{\gamma}_{k}$ is the average faded SNR of the $k$ th branch, the average number of combined branches is given again by (12), but $P_{\Gamma_{i}}(\cdot)$ is now given by the following compact form, presented in [18] as

$$
P_{\Gamma_{i}}(x)=\sum_{\substack{i_{1}, i_{2}, \ldots, i_{L}=1 \\ i_{1} \neq i_{2} \neq, \ldots, \neq i_{L}}}^{L}\left(\prod_{l=1}^{L} \frac{\bar{\gamma}_{i l}^{-1}}{\min (l, L)}\right) \times \sum_{j=1}^{L} \frac{C_{j}}{a_{j}}\left(1-e^{-a_{j} x}\right)
$$

where

$$
a_{j}=\frac{\sum_{k=1}^{L} \bar{\gamma}_{i l}^{-1}}{\min (j, L)}, C_{j}=\prod_{\substack{n=1 \\ n \neq j}}^{L}\left(a_{n}-a_{j}\right)^{-1}
$$

while $F_{l}(\mu)$ can be found in [17, eq. (12)].

\section{Numerical Results And Simulations}

In this section we present numerical results to demonstrate the advantage of the A-GSC receiver compared to MS-GSC and NT-GSC ones. We considered an uncoded system with binary phase shift keying (BPSK) modulation and a Rayleigh fading environment. The average number of combined branches is also verified by computer simulations, in order to show the validity of the presented mathematical analysis.

In Fig. 3 , the average bit error probability (ABEP) and $\bar{N}_{c}$ are plotted against the SNR of the first branch assuming two 

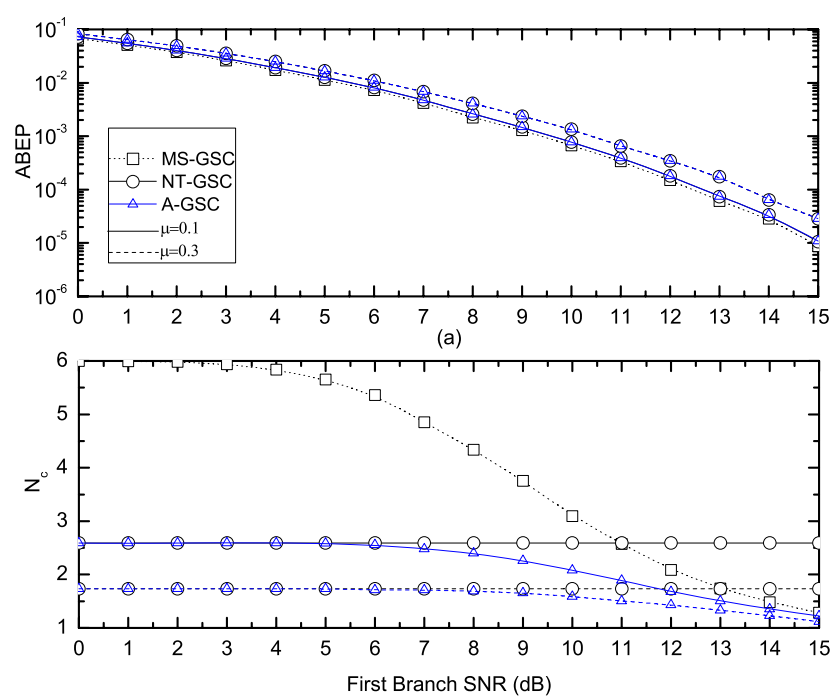

(b)

Fig. 4. ABEP and $\bar{N}_{c}$ with $L=6$ in i.n.i.d Rayleigh fading channels with exponential PDP $(d=1)$.

values for the threshold $\mu$, while $\gamma_{T}=10 \mathrm{~dB}$. For $\mu=0.1$ and small values of the SNR, MS-GSC uses almost all the available branches trying to reach the desired threshold $\gamma_{T}$, since the individual quality of each branch is ignored (i.e., the performance is independent of the threshold $\mu$ ). On the other hand, NT-GSC uses a steady number of branches ignoring the output SNR and as a result, for medium and high values of the SNR it utilizes unnecessarily many branches, while a sufficiently good quality could be achieved with less combined branches. Contrarily, A-GSC considers both the combined output SNR and the quality of each branch separately, resulting in an improved adaptability to channel conditions. For small SNR values, A-GSC avoids combining unnecessary weak branches, while for medium to high SNR regime it combines only those branches that are sufficient for the desired quality of communication. For $\mu=0.3$, the test per branch becomes stricter and the number of combined branches is reduced, yet at the cost of performance. The savings in power and computational resources are more important in i.n.i.d fading channels, as seen in Fig. 4, where an exponential PDP is assumed, i.e. $\bar{\gamma}_{k}=\bar{\gamma} e^{-d(k-1)}$, where $d$ is the decay factor. A-GSC uses less diversity branches than MS-GSC or NT-GSC by properly adapting to channel conditions.

In Fig. 5 the ABEP from computer simulations and $\bar{N}_{c}$ are plotted against the output threshold $\gamma_{T}$ (assuming that the SNR of the first branch is $5 \mathrm{~dB}$ ). We see that MS-GSC keeps adding branches even if no performance improvement is expected with the addition of an extra branch, in contrast to AGSC, which estimates whether the addition of another branch compensates for the expected performance improvement or not. Similar observations can be made in Fig. 6, where i.n.i.d fading channels are assumed.
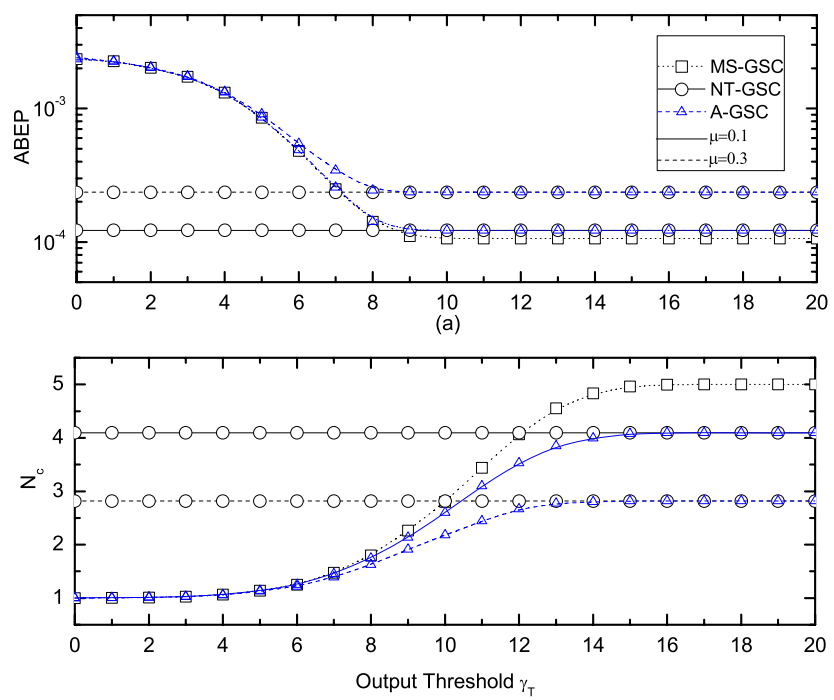

(b)

Fig. 5. ABEP and $\bar{N}_{c}$ with $L=5$, against the output threshold, in i.i.d Rayleigh fading channels.
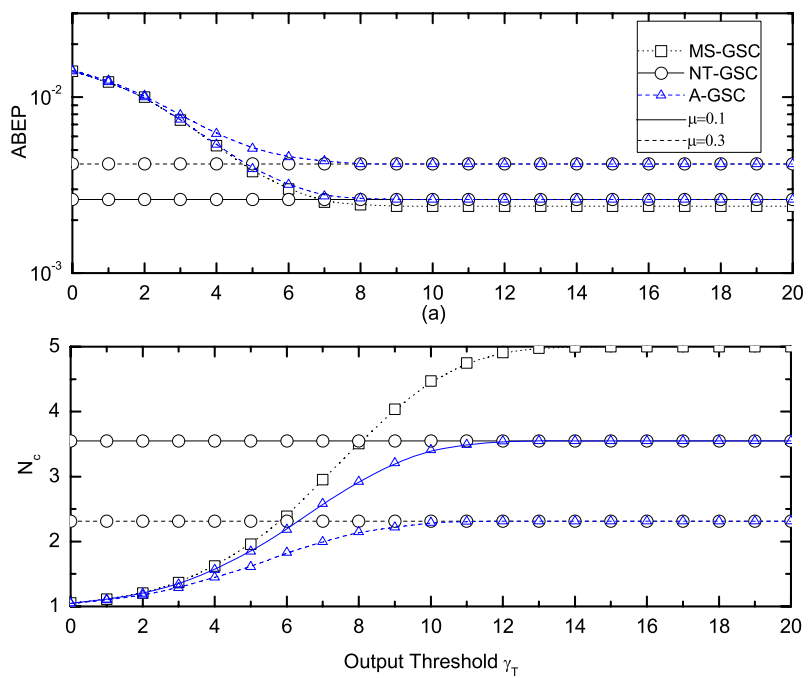

(b)

Fig. 6. ABEP and $\bar{N}_{c}$ with $L=5$, against the output threshold, in i.n.i.d Rayleigh fading channels with exponential PDP $(d=0.5)$.

\section{CONCLUSIONS}

An adaptive GSC receiver for diversity rich fading environments was presented and analyzed. By combining the features of the widely used MS-GSC (or MEC-GSC) and NTGSC receivers, A-GSC achieves better adaptation to channel conditions, without increasing the system's complexity. The suggested receiver compromises between the hardware complexity, power consumption and performance gain that each additional diversity branch induces, resulting in important savings in power and computational resources. 


\section{ACKNOWLEDGEMENT}

The authors would like to thank Mr. D. Michalopoulos, Aristotle University of Thessaloniki, for useful discussions on probability theory. Also, we would like to thank the Editor and the anonymous reviewers for their insightful comments and constructive suggestions that considerably improved the quality of this paper.

\section{REFERENCES}

[1] A. Annamalai, C. Tellambura, and V. K. Bhargava, "Efficient computation of MRC diversity performance in Nakagami fading channel with arbitrary parameters," Electron. Lett., vol. 34, no. 12, pp. 1189-1190, June 1998.

[2] V. A. Aalo, "Performance of maximal-ratio diversity systems in a correlated Nakagami-fading environment," IEEE Trans. Commun., vol. 43, no. 8, pp. 2360-2369, Aug. 1995.

[3] A. Annamalai, C. Tellambura, and V. K. Bhargava, "Exact evaluation of maximal-ratio and equal-gain diversity receivers for M-ary QAM on Nakagami fading channels," IEEE Trans. Commun., vol. 47, no. 9, pp. 1335-1344, Sept. 1999

[4] N. Kong, T. Eng, and L. B. Milstein, "A selection combining scheme for RAKE receivers," in Proc. IEEE Int. Conf. Univ. Personal Comm., Nov. 1995, pp. 426-429.

[5] M.-S. Alouini and M. K. Simon, "An MGF-based performance analysis of generalized selective combining over Rayleigh fading channels," IEEE Trans. Commun., vol. 48, pp. 401-415, Mar. 2000.

[6] R. K. Mallik and M. Z. Win, "Analysis of hybrid selection/maximalratio combining in correlated Nakagami fading," IEEE Trans. Commun., vol. 50, pp. 1372-1383, Aug. 2002.

[7] J. Foerster, Channel Modeling Subcommittee Report (Final), proposal vol. 1.0, IEEE P802.15.3a Working Group, 2003.

[8] A. I. Sulyman and M. Kousa, "Bit-error rate performance of a generalized diversity selection combining scheme in Nakagami fading channels," in Proc. IEEE Wireless Communications Networking Conf., Chicago, IL, Sept. 2000, pp. 1080-1085.
[9] X. D. Zhang and N. C. Beaulieu, "SER and outage of threshold based hybrid selection/maximal ratio combining over generalized fading channels," IEEE Trans. Commun., vol. 52, no. 12, pp. 2143-2153, Dec. 2004.

[10] L. Xiao and X. Dong, "Unified analysis of generalized selection combining with normalized threshold test per branch," IEEE Trans. Wireless Commun., vol. 5, no. 8, pp. 2153-2163, Aug. 2006.

[11] S. W. Kim, D. S. Ha, and J. H. Reed, "Minimum selection GSC and adaptive low-power RAKE combining scheme," in Proc. IEEE Int. Symp. Circuits Syst., May 2003, pp. 357-360.

[12] P. Gupta, N. Bansal, and R. K. Mallik, "Analysis of minimum selection H-S/MRC in Rayleigh fading," IEEE Trans. Commun., vol. 53, no. 5, pp. 780-784, May 2005.

[13] R. K. Mallik, P. Gupta, and Q. T. Zhang, "Minimum selection GSC in independent Rayleigh fading," IEEE Trans. Veh. Technol., vol. 54, no. 3, pp. 1013-1021, May 2005.

[14] H.-C. Yang, "New results on ordered statitics and analysis of minimumselection generalized selection combining (GSC)," IEEE Trans. Wireless Commun., vol. 5, no. 7, pp. 1876-1885, July 2006.

[15] H.-C. Yang and M.-S. Alouini, "MRC and GSC diversity combining with an output threshold," IEEE Trans. Veh. Technol., vol. 54, no. 3, pp. 10811090, May 2005.

[16] M.-S. Alouini and H.-C. Yang, "Minimum estimation and combining generalized selection combining (MEC-GSC)," IEEE Trans. Wireless Commun., vol. 6, pp. 526-532, Feb. 2007.

[17] A. Annamalai, G. Deora, and C. Tellambura, "Unified analysis of generalized selection diversity with normalized threshold test per branch," in Proc. IEEE Wirel. Commun. Netw. Conf., vol. 1, pp. 752-756. Mar. 2003.

[18] M. K. Simon and M.-S. Alouini, "A compact performance analysis of generalized selection combining (GSC) with independent but nonidentically distributed Rayleigh fading paths," IEEE Trans. Commun., vol. 50, pp. 1409-1412, Sept. 2002. 